\title{
Damage to the cuff of EMG tube at endotracheal intubation by using a lightwand -A case report-
}

\author{
Hyun-Sook Kim ${ }^{1}$, Keun-Suk Park ${ }^{1}$, Mae-Hwa Kang ${ }^{1}$, and Chong Doo Park ${ }^{2}$ \\ ${ }^{1}$ Department of Anesthesiology and Pain Medicine, Seoul National University Hospital, Seoul, ${ }^{2}$ Department of Anesthesiology, \\ Dongnam Institute of Radiological \& Medical Sciences, Busan, Korea
}

Electromyogpraphic endotracheal tube (EMG tube) is a new device used to monitor recurrent laryngeal nerve integrity during thyroid surgery. The EMG tube has 2 pairs of electrodes on the surface of silicon-based tube reached to inner space of tube cuff. We experienced an unusual endotracheal tube-related problem from the distinct structural feature of the EMG tube. In this case, we intubated a patient who had difficult airway with the EMG tube using a lightwand. After successful endotracheal intubation, we could not expand the pilot balloon and ventilate the patient effectively. We removed the EMG tube and found that one of electrodes of the EMG tube is bended and made a right angle with the long axis of the tube, and perforated the tube cuff. So we report this case to make anesthesia providers aware that much more attention is needed to use EMG tube during endotracheal intubation. (Korean J Anesthesiol 2010; 59: S17-S20)

Key Words: Complication, Electromyographic endotracheal tube, Intubation, Lightwand, Recurrent laryngeal nerve monitoring, Thyroid surgery.

To prevent damage to the recurrent laryngeal nerve, one of serious complications during thyroid surgery, intraoperative laryngeal electromyography monitoring can be conducted [1]. The electromyographic endotracheal tube (EMG tube), used to facilitate laryngeal electromyography monitoring, has two pairs of electrodes on the surface of silicon-based reinforced tube connected to inner space of the endotracheal tube cuff and is designed to monitor the vocalis muscle electromyography by the electrodes touching the vocalis muscle (Fig. 1). NIMResponse ${ }^{\circledR}$ Nerve Integrity Monitoring System (Medtronic, USA) is the commonly used device for laryngeal electromyographic monitoring.

We report our experience of a balloon cuff being damaged by the protrusion of electrodes attached to the EMG tube after performing intubation of the EMG tube with a lightwand for a patient with difficult airway, with review of the relevant literature.

Received: February 18, 2010. Revised: 1st, March 10, 2010; 2nd, March 21, 2010. Accepted: April 2, 2010.

Corresponding author: Chong Doo Park, M.D., Department of Anesthesiology, Dongnam Institute of Radiological \& Medical Sciences, 40, Jwadong-gil, Jangan-eup, Gijang-gun, Busan 619-953, Korea. Tel: 82-51-720-5062, Fax: 82-51-720-5959, E-mail: cdpark@snu.ac.kr

This was presented at the $86^{\text {th }}$ Annual Scientific Meeting of the Korean Society of Anesthesiologists, 2009, International Convention Center, Jeju, Korea. (c) This is an open-access article distributed under the terms of the Creative Commons Attribution Non-Commercial License (http:// creativecommons.org/licenses/by-nc/3.0/), which permits unrestricted non-commercial use, distribution, and reproduction in any medium, provided the original work is properly cited. 


\section{Case Report}

A 43-year-old, 70-kg, 167-cm man with thyroid cancer was admitted for robotic total thyroidectomy. He had no other underlying disorders and medical history of abnormalities. There were no abnormalities in preoperative hematological test, simple X-ray test, and ECG, except for abnormal finding of mild spondylosis shown in C-spine radiography.

Premedication was not provided. Before induction of anesthesia, the blood pressure was $144 / 79 \mathrm{mmHg}$, heart rate was $92 / \mathrm{min}$, and $\mathrm{O}_{2}$ saturation was $98 \%$. After performing denitrogenation with $100 \%$ oxygen through facial mask, the continuous infusion of remifentanil $4.5 \mathrm{ng} / \mathrm{ml}$ and propofol $5 \mu \mathrm{g} / \mathrm{ml}$ was initiated with using target controlled infusion system (Orchestra, Fresenius vial, France). Upon the patient's loss of consciousness, rocuronium $50 \mathrm{mg}$ was intravenously injected. After 3 minutes' mask ventilation, neck extension was tried to perform endotracheal intubation, but there was limitation in extension. Then, an intubation under direct laryngoscope was tried but failed because the vision of laryngoscope was Grade III of Cormack-Lehane classification of difficult laryngoscopy that only epiglottis may be seen. Next, a lightwand (Surch-Lite ${ }^{\mathrm{TM}}$ Orotracheal Lighted Intubation Stylet, Aaron) was used for intubation but this try was also unfruitful because the lightwand was not properly fixed to the EMG tube so that the lightwand was off the tip of the tube. At the second attempt, endotracheal intubation was achieved when the tip of the lightwand was bended up to $90^{\circ}$ at a point of $7.5 \mathrm{~cm}$ down from the top to keep

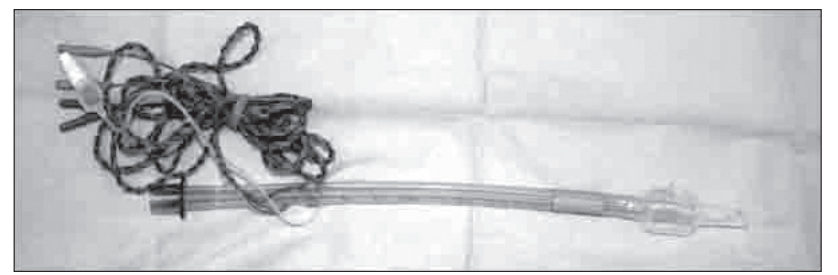

Fig. 1. Electromyographic tube (EMG tube) is a silicon-based reinforced tube and two pairs of electrodes are reaching to inner space of tube cuff along the surface.

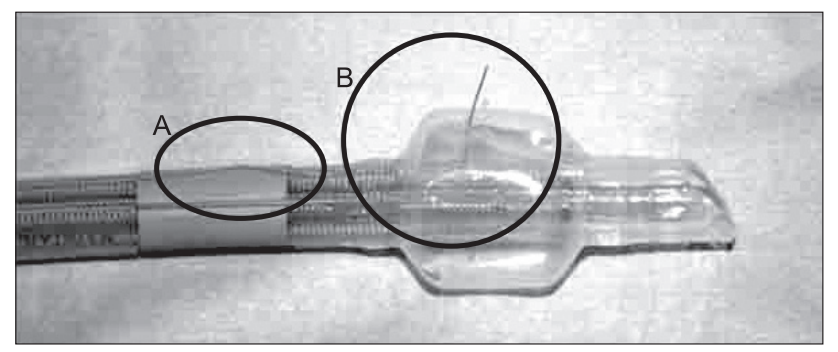

Fig. 2. (A) One of the EMG electrodes is detached from the surface of the tube at its bended spot. (B) The end of a detached EMG electrode has protruded out of the balloon. the lightwand from being loosened off. After intubation, a large amount of air was provided into the cuff but it failed to inflate the cuff and ended up with a leakage of the breathing circuit, by which we suspected of damaged cuff and replaced the EMG tube with a new one using a tube exchanger. On examining the replaced EMG tube, one of the four electrodes was found to be bent almost at 90 degree inside the cuff to have penetrated the cuff (Fig. 2). At endotracheal suction, a small amount of blood tinged secretion was aspirated, but no active bleeding was detected. Afterwards, anesthesia proceeded without further difficulty, and when post-operative endotracheal suction was performed, a small amount of blood tinged sputum was aspirated. To confirm any damage to the airway, a fiberoptic bronchoscope was inserted through the endotracheal tube and then, close examination revealed mild mucosal damaged area at the right bottom of the glottis, but neither clinically serous bleeding nor laceration trace was found (Fig. 3).

After his spontaneous breathing was restored, the patient was extubated and transferred to the recovery room. Without any development of abnormal symptoms at the recovery room, he was transported to the general ward. Afterwards, he complained of pain at the throat, but there were no abnormalities such as bleeding or respiration difficulty. On post-operative day 3 , he was discharged, when he stopped complaining of throat pain and no evidence of damage to the recurrent laryngeal nerve was detected.

\section{Discussion}

The use of EMG tube was initiated at the early 1990s to prevent damage to the recurrent laryngeal nerve (RNL) during

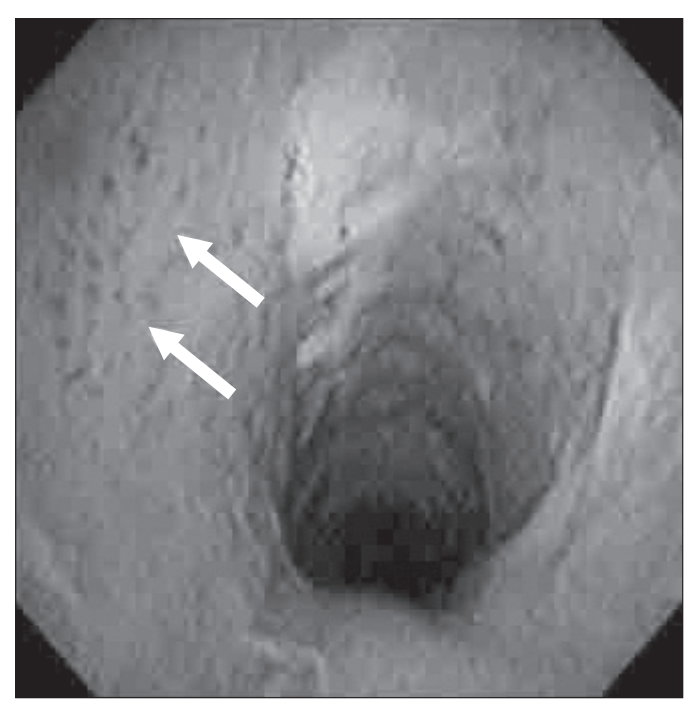

Fig. 3. Mild mucosal damage is observed (arrows) at just below vocal cord by a fiberoptic bronchoscope inspection. 
thyroidectomy. According to Horne et al. [2], 27\% of American otolaryngologists do laryngeal electromyography monitoring with EMG tube during thyroid gland surgery. In Korean, however, there has been no report about the prevalence of intraoperative recurrent laryngeal nerve monitoring, while our hospital currently are employing a RLN monitor for patients undergoing thyroidectomy with using robot.

EMG tube is a kind of reinforced tube made of soft siliconebase, in which arc-shape tube curve is not maintained as in polyvinyl chloride (PVC)-based one. When endotracheal intubation can be performed on patients of Grade I or II of Cormack \& Lehane classification, the use of stylet is not needed in PVC-based tube, but it is quite necessary if EMG tube is used. Such characteristic of soft silicon-based material differentiates EMG tube from PVC tube in many ways.

In the present case, the primary cause of electrode exposure of EMG tube results from the fact that the lightwand was used in extremely bent shape with almost perpendicular angle. Examination of the damaged EMG tube indicates that one of EMG electrodes was loosened off the tube surface and revealed a rift from the area contacting the vocal cords (blue part in the picture) (Fig. 2). We confirmed that the electrode was not herniated when being formed in curve before performing endotracheal intubation. It is suspected that the herniation of the electrode took place when the tube alone was extended as the lightwand was withdrawn and the tube was inserted simultaneously and that the intubation proceeded with the herniated electrode in contact with the tracheal inner wall so that the electrode turned bended at nearly $90^{\circ}$.

Then, what is the optimal angle and length of the lightwand when it is used at endotracheal intubation? In a prospective, randomized study by Nishiyama et al. [3], it is recommended that the lightwand should be bent in $40^{\circ}$ to $60^{\circ}$, with the extrusion of 1 to $2 \mathrm{~cm}$ from the tracheal tube. In a study of Chen et al. [4], they suggested that the proper bent length of the tube should differ according to patient's thyromental distance (TMD): bend between $6.5-8.5 \mathrm{~cm}$ length of the wand for patients having a TMD $>5.5 \mathrm{~cm}$; bend at $6.5 \mathrm{~cm}$ length of the wand for patients with a TMD $<5.5 \mathrm{~cm}$. In the current study, considering the suggestions above, the bent length seems to be appropriate while the angle excessively big. However, the result of study by Nishiyama et al., in which patients relatively easy for endotracheal intubation were selected, without Cormack \& Lehane Grade III and IV patients, is hard to apply to our patients with difficulty in endotracheal intubation with laryngoscope. To date only a few reports have explored the effective angle and length of the lightwand, and further study seems to be necessary to overcome the incidence of herniation of electrodes attached to the EMG tube surface at large angled bends.

Even in case of performing intubation using the direct laryngoscope with a stylet on patients with endotracheal intubation difficulty, sometimes the tube may be employed in about $90^{\circ}$ bent shape, when an electrode can be slipped off as in the case of lightwand as the tube is bent and extended.

The second cause of electrode exposure of EMG tube is that the tube was disinfected and reused. EMG tube is expensive (about $W 500,000$ ) and it is not covered by medical insurance because its eligibility for health insurance is still in the middle of examination at Health Insurance Review \& Assessment Service. In principle EMG tube is not reusable, but in fact, the tube is being reused 3 to 5 times through disinfection process in our hospital. The EMG tube used in the present study also was reused one after being disinfected once.

The dissection of the internal wall of reinforced endotracheal tube has been frequently reported in previous studies, in which frequent use and $\mathrm{N}_{2} \mathrm{O}$ diffusion were suspected for its cause [5,6]. In particular, Tose et al. [7] demonstrated that sequence of frequent disinfection and reuse can damage reinforced endotracheal tube even without use of $\mathrm{N}_{2} \mathrm{O}$, by their report of dissection of a reused reinforced endotracheal tube during total intravenous anesthesia. Thus, it could be reasonable to suspect of EMG tube deformation during disinfection process for the present case, too, but such possibility can be excluded because the EMG tube was confirmed to be in good condition before endotracheal intubation.

The instruction manual of EMG tube specifies that reuse is prohibited and disinfection may cause damage or breakdown of the tube. If reuse of EMG tube had caused complications due to infection, the anesthesiologist could not get away from the legal responsibility. When reuse of EMG tube comes to an unavoidable choice out of considering its expensive cost, however, full caution should be exercised to confirm the condition of the tube before performing endotracheal intubation and to check the dissection of the internal wall during its use. Above all, the coverage of EMG tube by medical insurance should be made as soon as possible.

To date, there have been two cases reported on complications related to EMG tube. Reporting a case of ventilation problem due to herniation of the EMG endotracheal tube cuff caused by $\mathrm{N}_{2} \mathrm{O}$ diffusion, Gleich et al. [8] observed that cuff herniation occurred in EMG tube at significantly lower pressure than in endotracheal tube made from PVC. Silicone membranes are so susceptible to diffusion of $\mathrm{N}_{2} \mathrm{O}$ [9] that when Gleich et al. performed 30 minutes of ventilation with the use of $70 \% \mathrm{~N}_{2} \mathrm{O}$, the cuff pressure increased in PVC tube by $10 \mathrm{mmHg}$ while in EMG tube by $50 \mathrm{mmHg}$. Cuff pressure greater than $30 \mathrm{cmH}_{2} \mathrm{O}$ (approximately $22 \mathrm{mmHg}$ ) compresses mucosal capillaries and impairs blood flow, so that total occlusion occurs when cuff pressure reaches over $50 \mathrm{cmH}_{2} \mathrm{O}(37 \mathrm{mmHg})$ [10], in which the intralaryngeal portion of the recurrent laryngeal nerve 
may be damaged. Therefore, it is advised that the use of $\mathrm{N}_{2} \mathrm{O}$ should be avoided in case of using EMG tube. It is customary in clinic practice to assess intracuff pressure by palpation of the pilot balloon, but it is recommended to use the manometer for accurate measurement because the pilot balloon of EMG tube has a much softer feel than that of PVC one and it does not provide a sensitive palpation for the accurate assessment even when the cuff pressure is increased enough with a large amount of air. In a study by Evanina and Hanisak [11] reporting that occlusion of EMG tube impeded endotracheal suctioning at the intensive care unit after operation, the authors recommended that endotracheal tube must be replaced in case of maintaining the endotracheal tube after operation.

Even though EMG tube is not widely used yet, it can be used not only to identify the recurrent laryngeal nerve during thyroidectomy, but also to monitor the vagus nerve during head and neck surgery [12]. Especially once the use of EMG tube is covered by medical insurance, the frequency of its use is expected to increase significantly. Therefore, anesthesiologists should be aware of the characteristics of EMG tube and its potential complications, and be well prepared to prevent complications.

\section{References}

1. Rice DH, Cone-Wesson B. Intraoperative recurrent laryngeal nerve monitoring. Otolaryngol Head Neck Surg 1991; 105: 372-5.

2. Horne SK, Gal TJ, Brennan JA. Prevalence and patterns of intraoperative nerve monitoring for thyroidectomy. Otolaryngol Head Neck
Surg 2007; 136: 952-6.

3. Nishiyama T, Matsukawa T, Hanaoka K. Optimal length and angle of a new lightwand device (Trachlight). J Clin Anesth 1999; 11: 3325.

4. Chen TH, Tsai SK, Lin CJ, Lu CW, Tsai TP, Sun WZ. Does the suggested lightwand bent length fit every patient? The relation between bent length and patient's thyroid prominence-tomandibular angle distance. Anesthesiology 2003; 98: 1070-6.

5. Rao GS, Ali Z, Ramkiran S, Chandrasekhar HS. The dissection of a reinforced endotracheal tube causing near-fatal intraoperative airway obstruction. Anesth Analg 2006; 103: 1624-5.

6. Jeon YS, Kim YS, Joo JD, Kang EG, In JH, Choi JW, et al. Partial airway obstruction caused by dissection of a reinforced endotracheal tube. Eur J Anaesthesiol 2007; 24: 983-4.

7. Tose R, Kubota T, Hirota K, Sakai T, Ishihara H, Matsuki A. Obstruction of an reinforced endotracheal tube due to dissection of internal tube wall during total intravenous anesthesia. Masui 2003; 52: 1218-20.

8. Gleich SJ, Nicholson WT, Jacobs TM, Hofer RE, Sprung J. Inability to ventilate while using a silicone-based endotracheal tube. J Clin Anesth 2008; 20: 389-92.

9. Maino P, Dullenkopf A, Bernet V, Weiss M. Nitrous oxide diffusion into the cuffs of disposable laryngeal mask airways. Anaesthesia 2005; 60: 278-82.

10. Seegobin RD, van Hasselt GL. Endotracheal cuff pressure and tracheal mucosal blood flow: endoscopic study of effects of four large volume cuffs. Br Med J (Clin Res Ed) 1984; 288: 965-8.

11. Evanina EY, Hanisak JL. Case study involving suctioning of an electromyographic endotracheal tube. AANA J 2005; 73: 111-3.

12. Ito H, Sobue K, So M, Hirate H, Sugiura T, Azami T, et al. Intraoperative monitoring of vagal nerve activity with wire electrodes. Acta Anaesthesiol Scand 2006; 50: 1304-5. 